\title{
Cigarette smoking and response to inhaled corticosteroids in COPD
}

\author{
To the Editor:
}

Inhaled corticosteroids (ICS) and long-acting $\beta$-agonists (LABA) are frequently used in patients with chronic obstructive pulmonary disease (COPD). Combination treatment with ICS/LABA improves lung function and quality of life, and reduces exacerbation frequency compared to treatment with either ICS or LABA alone. Although it is presumed that continued cigarette smoking impairs acute responses to these medications in patients with COPD, there is little direct evidence to support this view. Studies of ICS use in asthma have shown less short-term improvement in lung function and reduced anti-inflammatory effects in active smokers compared to non-smokers [1,2]. Although similar effects are plausible in COPD, they have not been definitively demonstrated. We hypothesised that former smokers with COPD would have greater short- and long-term changes in lung function, respiratory-related quality of life, and exacerbation risk, in response to ICS than continuing and intermittent smokers.

We used data from a large double-blind, randomised, controlled trial, comparing the effects of combination ICS (fluticasone furoate, FF) and LABA (vilanterol, VI), with ICS (FF) alone, LABA (VI) alone, and a placebo (the Study to Understand Mortality and Morbidity in COPD (SUMMIT) trial). We performed a post hoc analysis to assess the relationship between smoking status and respiratory outcomes. The details of SUMMIT have been previously published [3]. Briefly, participants were 40-80 years of age, with at least a 10 pack-year smoking history, and post-bronchodilator $\mathrm{FEV} 1 / \mathrm{FVC} \leqslant 0.70$ with a post-bronchodilator FEV1 50\%-70\% predicted, and enhanced cardiovascular risk. Spirometry assessments were made at enrolment and every 3 months thereafter, and respiratory-related health status assessments (St George's Respiratory Questionnaire (SGRQ)) were measured in a subset, at enrolment and at 3, 6 and every 6 months thereafter. Exacerbations were defined as the worsening of respiratory status, necessitating therapy with antibiotics and/or systemic corticosteroids, and those that required hospitalisation were deemed severe exacerbations. Participants were classified as either current or former smokers, based on smoking status at enrolment. The primary outcome was change in post-bronchodilator FEV1 at each visit, compared to baseline, up to 1 year after enrolment, and secondary outcomes were change in SGRQ up to 1 year after enrolment and exacerbation risk over the entire study. All analyses for outcomes between current and former smokers were performed in comparison to changes in the placebo group. The FEV 1 and SGRQ were analysed using mixed model repeated measures. The models included terms for treatment, age, gender, baseline measurement, visit, smoking status, baseline measurement by visit, treatment by visit, smoking status by visit, smoking status by treatment and smoking status by treatment by visit. Exacerbation rates were analysed using negative binomial regression. The model included terms for treatment, age, gender, number of previous COPD exacerbations in the 12 months prior to screening, smoking status and smoking status by treatment. No adjustments were made for multiple comparisons.

Of the 16485 participants, 7678 (47\%) were current smokers at enrolment, and 8807 (53\%) were former smokers. Current smokers included $27 \%$ females, $63 \pm 8$ (mean \pm SD) years of age, and body mass index (BMI) of $27 \pm 6 \mathrm{~kg} \cdot \mathrm{m}^{-2}$, whereas former smokers included $25 \%$ females, $67 \pm 7$ years of age and BMI $29 \pm 6 \mathrm{~kg} \cdot \mathrm{m}^{-2}$. During the course of the study, 1859 (11\%) participants quit smoking, $81(<1 \%)$ switched from non-smoker to active smoking status, $778(5 \%)$ had multiple changes in their smoking status, and 13767 (84\%) had no change in smoking status.

Compared with the placebo, the adjusted mean change in FEV1 at 3, 6, 9 and 12 months was greater in former smokers than in current smokers (figure 1), with both FF (mean difference 30, 95\% CI 9-51 mL; $\mathrm{p}=0.005$ ) and FF/VI (mean difference 22, 95\% CI 1-43 mL; $\mathrm{p}=0.038$ ), but not with VI alone (not shown,

@ERSpublications

Current smokers have a blunted FEV1 response and exacerbation reduction with inhaled corticosteroids http://ow.ly/PBIW30hcISK

Cite this article as: Bhatt SP, Anderson JA, Brook RD, et al. Cigarette smoking and response to inhaled corticosteroids in COPD. Eur Respir J 2018; 51: 1701393 [https://doi.org/10.1183/13993003.01393-2017]. 


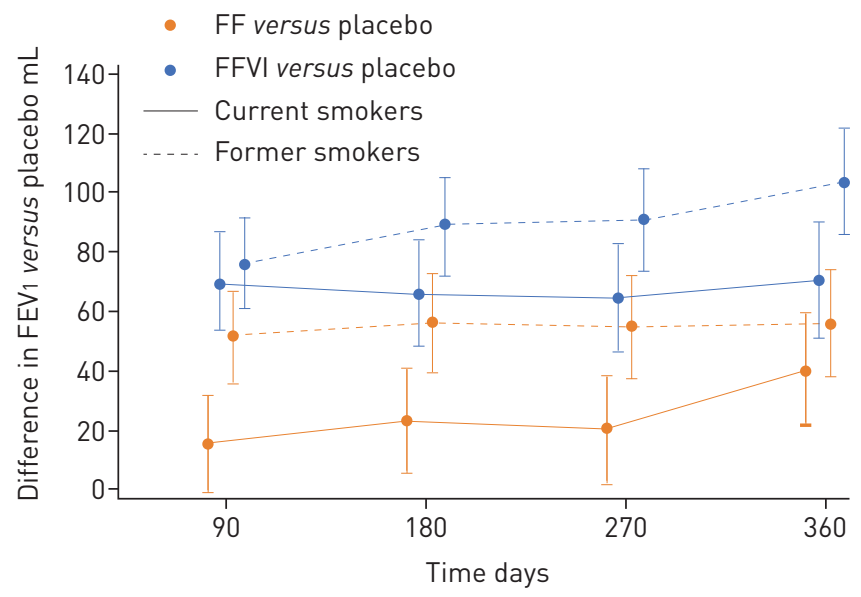

FIGURE 1 Comparison of change in forced expiratory volume in $1 \mathrm{~s}$ (FEV 1 ) at 90, 180, 270 and 360 days. Compared to current smokers, former smokers showed a greater response to active treatment with both fluticasone furoate (FF) (mean difference $30,95 \% \mathrm{Cl} 9-51 \mathrm{~mL} ; \mathrm{p}=0.005$ ) and FF/vilanterol (FF/VI) (mean difference $22,95 \% \mathrm{Cl} 1-43 \mathrm{~mL} ; \mathrm{p}=0.038$ ), adjusted for age, sex, baseline FEV1, visit, smoking status, FEV1 by visit, smoking status by visit, smoking status by treatment and interaction for smoking status by treatment by visit.

mean difference $-6,95 \% \mathrm{CI}-27$ to $15 \mathrm{~mL} ; \mathrm{p}=0.572$ ). For the SGRQ, the treatment differences associated with FF versus the placebo were similar, regardless of smoking status (mean difference $-1.0,95 \% \mathrm{CI}-2.8$ to 0.8 units; $\mathrm{p}=0.267$ ), and for FF/VI versus the placebo (mean difference $-1.1,95 \% \mathrm{CI}-2.9$ to 0.7 units; $\mathrm{p}=0.226$ ). However, for VI versus the placebo, there was greater benefit for former smokers than for current smokers (mean difference $-2.5,95 \% \mathrm{CI}-4.3$ to -0.8 units; $\mathrm{p}=0.005$ ). In addition, former smokers had a greater percentage reduction in rates of moderate or severe exacerbations when on FF/VI compared to the placebo than did current smokers (36\%, 95\% CI 27\%-43\% versus $19 \%, 95 \%$ CI $7 \%-29 \%$; $=0.013$ ). No difference in exacerbation rate reduction was observed between former versus current smokers taking FF versus the placebo $(17 \%, 95 \%$ CI $6 \%-26 \%$ versus $6 \%, 95 \%$ CI $-8 \%$ to $17 \%$; $\mathrm{p}=0.176)$, or taking VI versus the placebo $(14 \%, 95 \%$ CI $4 \%-24 \%$ versus $4 \%$, $95 \%$ CI $-9 \%$ to $16 \% ; \mathrm{p}=0.227)$. No differences in hospitalised exacerbation rate reduction were observed among former versus current smokers taking FF/VI versus the placebo $(14 \%, 95 \% \mathrm{CI}-22 \%$ to $40 \%$; $\mathrm{p}=0.396)$, or $\mathrm{FF}$ versus the placebo $(-16 \%, 95 \% \mathrm{CI}-64 \%$ to $18 \%$; $\mathrm{p}=0.413)$, or VI versus the placebo $(4 \%, 95 \% \mathrm{CI}-35 \%$ to $32 \%$; $\mathrm{p}=0.806)$. There was no evidence to suggest that smoking status influenced the risk of pneumonia associated with treatment (the overall p-value was 0.960 for smoking by treatment for all groups, and 0.775 for FF versus the placebo, 0.584 for FF/VI versus the placebo and 0.802 for VI versus the placebo). After adjustment for age, sex, smoking status and treatment by smoking status interaction, the hazard ratios for pneumonia in current and former smokers, comparing FF/VI versus the placebo, were 1.10 , 95\% CI $0.81-1.50$ and 0.99 , 95\% CI $0.79-1.25$, respectively; in FF compared with the placebo, $1.0795 \%$ CI $0.79-1.45$ and $1.01,95 \%$ CI $0.80-1.28$, respectively; and in VI compared with the placebo, $0.75,95 \%$ CI $0.53-1.04$ and $0.71,95 \%$ CI $0.55-0.91$, respectively.

We demonstrated that compared to former smokers, current smokers have a blunted FEV1 response with ICS and a smaller reduction in exacerbation frequency with ICS/LABA. Data on continued smoking and response to therapy are mostly derived from studies in asthma. In a small study of patients with mild-to-moderate asthma, continuing smokers had less improvement in FEV1/FVC at 6 months with budesonide compared to non-smokers [4]. Chalmers et al. [1] also found that in mild asthma, non-smokers had a significantly greater increase in morning peak expiratory flow (PEF) after 3 weeks of fluticasone therapy than did continuing smokers. Two randomised controlled trials have confirmed that continued smoking is associated with a lower improvement in PEF [2, 5]. To the best of our knowledge, these assessments have not been made for ICS or ICS/LABA in COPD, and studies of the impact of smoking on responses to other COPD medications have shown varied results. MoITA et al. [6] found no difference in FEV1 response by smoking status, but analyses of the UPLIFT study showed that short- and long-term improvement in FEV1 with tiotropium was greater in continuing smokers than in ex-smokers or intermittent smokers [7]. Post hoc analyses suggest that the exacerbation reduction effect of azithromycin might not be seen in those who actively smoke [8].

The mechanisms underlying the blunted response to these medications in active smokers are unknown, although the pro-inflammatory effects of cigarette smoke have been well described and could blunt the anti-inflammatory effects of ICS [9]. This is supported by our finding of no difference in response for FEV1 and exacerbations by smoking status with VI alone. Alternatively, the poor response could be due to relative steroid resistance mediated by a reduction in the enzyme histone deacetylase 2 , which is essential 
for glucocorticoid binding to their receptors [9]. We found no similar differences in change in the SGRQ, likely because it reflects outcomes with more heterogeneous mechanisms.

One limitation of the present study is our combination of intermittent and continued smokers into one group, based on smoking status at enrolment. However, previous studies on lung function and exacerbations suggest that continuing and intermittent smokers behave similarly in terms of lung function decline, as well as exacerbation frequency $[10,11]$. Active smoking status was self-reported. In addition, smoking status changed in a small proportion of patients over the course of the study. Some exacerbations might have gone unreported, but these are likely to occur across all groups of the study. Our findings have considerable public health importance, as 39\% of all patients with COPD continue to smoke, and smoking cessation could result in better overall outcomes [12].

In summary, similar to the effects observed in patients with asthma, continued smoking is associated with an impaired response to ICS and thereby affects the attainment of important clinical outcomes in COPD patients.

Surya P. Bhatt ${ }^{1}$, Julie A. Anderson ${ }^{2}$, Robert D. Brook ${ }^{3}$, Peter M.A. Calverley ${ }^{4}$, Bartolome R. Celli ${ }^{5}$, Nicholas J. Cowans ${ }^{6}$, Courtney $\mathrm{Crim}^{7}$, Fernando J. Martinez ${ }^{8}$, David E. Newby ${ }^{9}$, Jørgen Vestbo ${ }^{10}$, Julie C. Yates and Mark T. Dransfield ${ }^{1,11}$

${ }^{1}$ Division of Pulmonary, Allergy and Critical Care Medicine and Lung Health Center, University of Alabama at Birmingham, Birmingham, AL, USA. ${ }^{2}$ GlaxoSmithKline, Stockley Park, UK. ${ }^{3}$ Division of Cardiovascular Medicine, University of Michigan, Ann Arbor, MI, USA. ${ }^{4}$ School of Ageing and Chronic Disease, University of Liverpool, Liverpool, UK. ${ }^{5}$ Division of Pulmonary and Critical Care Medicine, Brigham and Women's Hospital, Boston, MA, USA. ${ }^{6}$ Veramed, Twickenham, UK. ${ }^{7}$ GlaxoSmithKline, Research Triangle Park, USA. ${ }^{8}$ Division of Pulmonary and Critical Care Medicine, Weill Cornell Medical College of Cornell University, New York, NY, USA. ${ }^{9}$ Division of Cardiology, University of Edinburgh,

Edinburgh, UK. ${ }^{10}$ Division of Infection, Immunity and Respiratory Medicine, University of Manchester, Manchester, UK.

${ }^{11}$ Division of Pulmonary and Critical Care Medicine, Birmingham VA Medical Center, Birmingham, AL, USA.

Correspondence: Surya P. Bhatt, University of Alabama at Birmingham, Division of Pulmonary, Allergy and Critical Care Medicine, THT 422, 1720, 2nd Avenue South, Birmingham, AL 35294, USA. E-mail: sbhatt@uabmc.edu

Received: July 122017 | Accepted after revision: Oct 252017

Acknowledgements

Author contributions were as follows. Study design: S.P. Bhatt and M.T. Dransfield. Statistical analyses: J.A. Anderson and N.J. Cowans. Data interpretation: S.P. Bhatt and M.T. Dransfield. Manuscript writing: S.P. Bhatt and M.T. Dransfield. Critical review of the manuscript for important content: all authors.

This study is registered at ClinicalTrials.gov with identifier NCT01313676.

Support statement: The study was funded by GSK Study 113782 (NCT01313676). Funding information for this article has been deposited with the Crossref Funder Registry.

Conflict of interest: Disclosures can be found alongside this article at erj.ersjournals.com

\section{References}

1 Chalmers GW, Macleod KJ, Little SA, et al. Influence of cigarette smoking on inhaled corticosteroid treatment in mild asthma. Thorax 2002; 57: 226-230.

2 Lazarus SC, Chinchilli VM, Rollings NJ, et al. Smoking affects response to inhaled corticosteroids or leukotriene receptor antagonists in asthma. Am J Respir Crit Care Med 2007; 175: 783-790.

3 Vestbo J, Anderson JA, Brook RD, et al. Fluticasone furoate and vilanterol and survival in chronic obstructive pulmonary disease with heightened cardiovascular risk (SUMMIT): a double-blind randomised controlled trial. Lancet 2016; 387: 1817-1826.

4 Shimoda T, Obase Y, Kishikawa R, et al. Influence of cigarette smoking on airway inflammation and inhaled corticosteroid treatment in patients with asthma. Allergy Asthma Proc 2016; 37: 50-58.

5 Tomlinson JE, McMahon AD, Chaudhuri R, et al. Efficacy of low and high dose inhaled corticosteroid in smokers versus non-smokers with mild asthma. Thorax 2005; 60: 282-287.

6 Moita J, Barbara C, Cardoso J, et al. Tiotropium improves FEV1 in patients with COPD irrespective of smoking status. Pulm Pharmacol Ther 2008; 21: 146-151.

7 Tashkin DP, Celli B, Kesten S, et al. Long-term efficacy of tiotropium in relation to smoking status in the UPLIFT trial. Eur Respir J 2010; 35: 287-294.

8 Han MK, Tayob N, Murray S, et al. Predictors of chronic obstructive pulmonary disease exacerbation reduction in response to daily azithromycin therapy. Am J Respir Crit Care Med 2014; 189: 1503-1508.

9 Tamimi A, Serdarevic D, Hanania NA. The effects of cigarette smoke on airway inflammation in asthma and COPD: therapeutic implications. Respir Med 2012; 106: 319-328.

10 Anthonisen NR, Connett JE, Murray RP. Smoking and lung function of Lung Health Study participants after 11 years. Am J Respir Crit Care Med 2002; 166: 675-679.

$11 \mathrm{Au}$ DH, Bryson CL, Chien JW, et al. The effects of smoking cessation on the risk of chronic obstructive pulmonary disease exacerbations. J Gen Intern Med 2009; 24: 457-463.

12 Centers for Disease Control and Prevention. Chronic obstructive pulmonary disease among adults - United States, 2011. MMWR Morb Mortal Wkly Rep 2012; 61: 938-943. 